\title{
Research on comprehensive stiffness characteristics of angular contact ball bearings under multi-factor coupling condition
}

\author{
Chunli LEI*, Pan CUI*, Pengyao CAO*, Kai LIU* and Ruizhe SONG* \\ *School of Mechanical and Electrical Engineering, Lanzhou University of Technology, \\ Lanzhou, 730050, China \\ E-mail: 2357489131@qq.com
}

Received: 29 May 2021; Revised: 17 September 2021; Accepted: 11 October 2021

\begin{abstract}
In order to research the dynamic performance of angular contact ball bearings of high-speed motorized spindles in detail, based on Hertz contact theory and bearing quasi-static model, considering the influence of centrifugal force, gyroscope moment and thermal effect on bearing operation, the modified quasi-static model is established to calculate the bearing contact stiffness. Then, considering the influence of temperature on the dynamic viscosity of lubricating oil, film stiffness model of bearing is also presented. On the basis, the bearing comprehensive stiffness model is proposed, and the influences of combined load and rotational speed on the bearing stiffness are investigated. The results show that as the speed increases, the radial contact stiffness increases, while the axial and angular contact stiffness decrease, the dynamic viscosity of the lubricating oil decreases, the film stiffness decreases and the comprehensive radial, axial and angular stiffness decrease. With the increase of axial load, the radial, axial and angular contact stiffness increase, the comprehensive stiffness increases. When the radial load increases, the radial, axial and angular contact stiffness decreases, while the comprehensive stiffness increases. It is found that the comprehensive stiffness considering the temperature is larger than without consideration of the temperature.
\end{abstract}

Keywords : Angular contact ball bearings, Comprehensive stiffness, Combined load, Thermal effect, Dynamic viscosity

\section{Introduction}

Motorized spindle is a new technology that integrates spindle and motor in the field of CNC (Computer Numerical Control) machine tools, and it is the core component of high-speed machine tools. Bearings are the supporting parts of motorized spindle, which directly determine the performance, processing quality and reliability of motorized spindle. Rolling bearings is widely used because of high rigidity, wide range of load and speed, and high working precision and transmission efficiency. The stiffness of the bearing reflects the main performance of motorized spindle system. Therefore, the accurate calculation of bearing stiffness is the primary task to analyze the dynamic characteristics of motorized spindle. Many scholars have conducted a lot of researches on the stiffness of rolling bearings. Jones $(1960,1966,1978)$ established the quasi-static model of rolling bearings to analyze the influence of centrifugal force and gyroscopic moment on the stiffness of the bearing according to the raceway control theory. Harris $(1973,1984)$ presented a bearing model which considered the influence of the elastohydrodynamic lubrication (EHL). Gupta (1984) considered the influence of the ball movement, stress state with the interaction between the various components on the bearing dynamic characteristics in his model. Hagiu et al. (1997) considered the film extrusion squeeze effect and elastic deformation of Hertzian contact, and studied the stiffness of high-speed angular contact ball bearing. Tang (2005) combined the film stiffness with contact stiffness to derive the comprehensive stiffness of the rolling bearing. Ali et al. (2010) studied the effect of different parameters on bearing stiffness through experiments. Fawzi et al. (1984) solved the nonlinear stiffness of 5-DOF angular contact ball bearing by Newton iteration method. Dougdag et al. (2012) carried out experimental verification on the model of nonlinear stiffness in dynamic and static modes. Noel et al. (2013) proposed a new stiffness matrix calculation method based on the dynamic model of rolling bearing. Huang et al. (2017) constructed a quasi-static model of angular contact ball bearings considering the effect of centrifugal expansion, then studied the influence of spindle speed and preload on the stiffness of bearings, but the 
complex working conditions were not taken into account. Zhang et al. (2017) proposed a new iterative algorithm on the basis of Jones' quasi-static model and stiffness analysis model and calculated the preload and stiffness of composite bearings. Yang et al. (2018) constructed the 5-DOF stiffness matrix is constructed based on the quasi-static model of angular contact ball bearings, developed a method to analyze various spindle stiffnesses with different configurations of bearing. Zhang et al. (2020) also carried out modeling and characteristic analysis for ball bearings under incomplete contact state, proposed new assumptions about initial position, and established a general analysis model for high-speed ball bearings under different load conditions.

However, according to the characteristics of high-speed angular contact ball bearings, the research on bearing stiffness is still insufficient now. Most researchers simply attribute the bearing stiffness to the contact stiffness calculated by the mechanical model, and the influence of lubricant on the bearing operation under the actual working conditions is ignored. Only a few researchers considered the contact stiffness and film stiffness of the bearing to obtain the overall stiffness. Xiong et al. (2015) put forward a bearing quasi-static modified model taking into account ferrule deformation and EHL to study the influence law of bearing dynamic stiffness. Lei et al. (2020) proposed a bearing comprehensive stiffness model under the condition of EHL, considering the centrifugal deformation and thermal deformation of the bearing. However, in these literatures, the temperature rise of each part of the bearing is unconsidered when the bearing is subjected to combined radial and axial load under the condition of EHL, and the influence of temperature rise on the contact stiffness, film stiffness and comprehensive stiffness of the bearing is not regarded. In this paper, based on the Jones' quasi-static model, considering the centrifugal force, gyroscopic moment and thermal deformation, a modified quasi-static model of bearing is established, and the Newton-Raphson method is adopted to calculate the dynamic parameters. The presented model is verified by the literature result and the influences of combined load and speed on the bearing stiffness are analyzed. At the same time, considering the influence of the temperature on the dynamic viscosity of the lubricating oil, the film stiffness model is constructed. On this basis, a calculation method for the comprehensive stiffness of the high-speed angular contact ball bearing is proposed, and the influences of the combined load and speed on the comprehensive stiffness are investigated. The result can provide a theoretical basis for the dynamics research and structure optimization of the rolling bearing.

\section{Modified quasi-static model of angular contact ball bearings}

The basic structure of angular contact ball bearing is shown in Figure 1(Li et al., 2020). $\alpha_{i}$ and $\alpha_{o}$ are the contact angle of the inner ring and outer ring, $r_{i}$ and $r_{o}$ are the raceway groove curvature radius of inner ring and outer ring respectively, $d_{m}$ is the bearing pitch diameter, $D_{b}$ is the diameter of the rolling element.

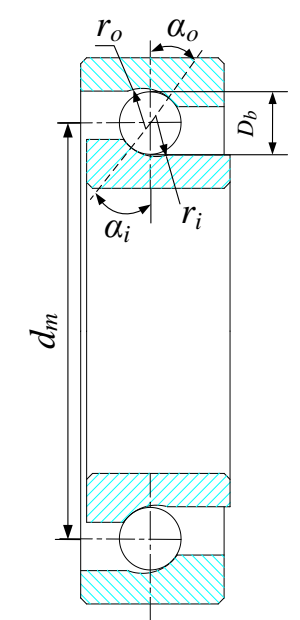

Fig. 1 Basic mechanism of angular contact bearing.

Considering the centrifugal force, gyroscope moment and thermal deformation of the angular contact ball bearing at high speed, a modified quasi-static model of the bearing is established, and then the contact stiffness of the bearing is calculated. 


\subsection{Thermal deformation of bearing parts}

When the bearing is running at high speed, frictional heat is generated by the relative movement between the various parts of the bearing, which increases the temperature of bearing parts, and the axial and radial thermal displacement of the inner and outer rings are produced respectively, which seriously affects the dynamic performance of the bearing. The thermal deformation can be written as follows (Harris,2001):

$$
u=a D_{T} \Delta T
$$

where, $u$ is the thermal deformation, $a$ is the thermal expansion coefficient, $\Delta T$ is the temperature rise, $D_{T}$ is the diameter of the part.

According to Eq. (1), the thermal deformation of the inner ring can be calculated:

$$
u_{i}=a_{i} D_{i} \Delta T_{i}
$$

where, $u_{i}$ is the thermal deformation of the inner ring, $a_{i}$ is the thermal expansion coefficient of the inner ring, $\Delta T_{i}$ is the temperature rise of the inner ring, $D_{i}$ is the diameter of the inner ring.

Similarly, the thermal deformation of the outer ring is:

$$
u_{o}=a_{o} D_{o} \Delta T_{o}
$$

where, $u_{o}$ is the thermal deformation of the outer ring, $a_{o}$ is the thermal expansion coefficient of the outer ring, $\Delta T_{\mathrm{o}}$ is the temperature rise of the outer ring, $D_{o}$ is the diameter of the outer ring.

The thermal deformation of the rolling elements is:

$$
u_{b}=a_{b} D_{b} \Delta T_{b}
$$

where, $u_{b}$ is the thermal deformation of the rolling element, $a_{b}$ is the thermal expansion coefficient of the rolling element, $\Delta T_{b}$ is the temperature rise of the rolling element.

From Eq. (2) to (4), it could be seen that the radial deformation $u_{r}$ caused by temperature rise is deduced as follows:

$$
u_{r}=u_{i}-2 u_{b}-u_{o}
$$

The axial deformation of the bearing is related to its configuration mode, and the axial deformation produced is different when the configuration method is different. Therefore, the axial deformation $u_{a}$ caused by the temperature rise can be expressed as:

$$
u_{a}= \pm \frac{a_{h} L_{h} \Delta T_{h}-a_{s} L_{s} \Delta T_{s}}{2}
$$

where, $a_{h}, a_{s}$ are the thermal expansion coefficients of the bearing housing and the shaft respectively. $L_{h}, L_{s}$ are the effective contact lengths between the bearing and the bearing housing, and the shaft and the bearing respectively. $\Delta T_{h}$, $\Delta T_{s}$ is the temperature rise of bearing housing and shaft respectively. Take "+" when double "O" is configured, Take "- 
" when double "X" is configured (Xiong et al., 2015).

\subsection{Force and motion analysis}

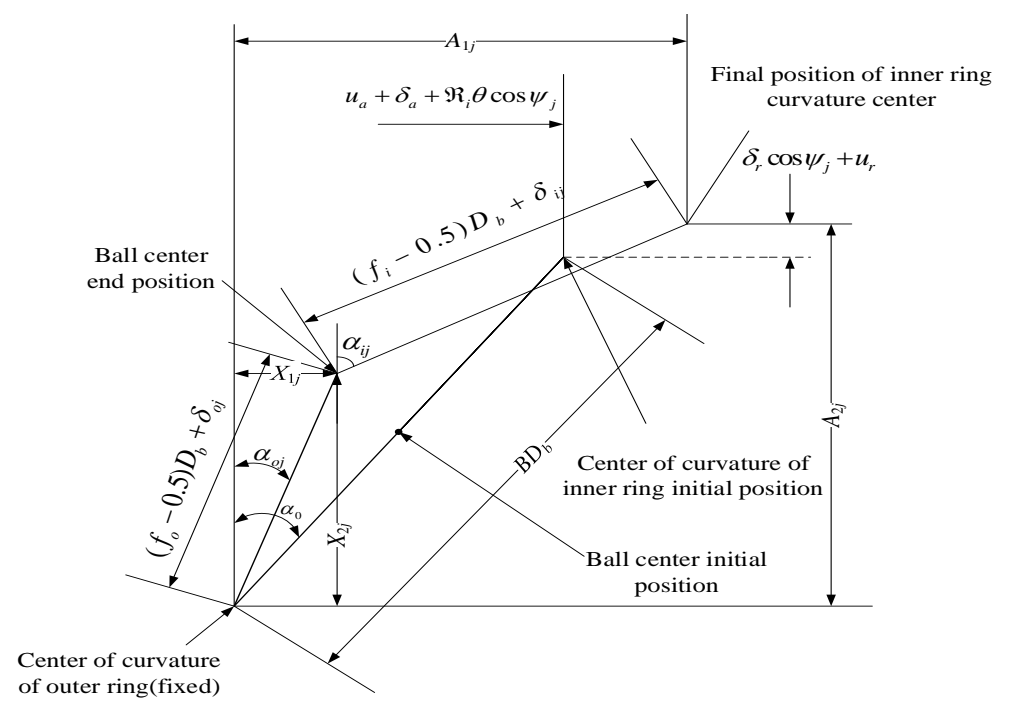

Fig. 2 Change of displacement of ball bearing considering thermal deformation

Figure. 2 shows the rolling element (the $j$-th ball) at any position of the rolling bearing, the positional relationship between the ball center and the curvature center of the inner and outer rings. $\alpha_{0}$ is the original contact angle, $\alpha_{i j}$ and $\alpha_{o j}$ are the contact angles of the inner and outer rings of the $j$-th rolling element, respectively, $\delta_{a}$ is the relative axial displacement of the inner and outer ring, $\delta_{r}$ is the relative radial displacement of the ring centers, $\theta$ is the relative angular displacement, $\psi_{j}$ is the azimuth of the $j$-th ball, $\mathfrak{R}_{i}$ is the radius of the locus of inner ring groove curvature centers, $A_{l j}$ and $A_{2 j}$ are the axial and radial displacements between the loci of the inner and outer ring groove curvature centers respectively, $D_{b}$ is rolling element diameter.

Considering the effect of the thermal deformation, at any position, the axial distance $A_{l j}$ and the radial distance $A_{2 j}$ between the loci of inner and outer raceway groove curvature centers at any ball position is written as:

$$
\begin{aligned}
& A_{l j}=B D_{b} \sin \alpha_{0}+\delta_{\alpha}+\Re_{i} \theta \cos \psi_{j}+u_{a} \\
& A_{2 j}=B D_{b} \cos \alpha_{0}+\delta_{r} \cos \psi_{j}+u_{r}
\end{aligned}
$$

where,

$$
\begin{aligned}
& B=f_{i}+f_{o}-1 \\
& \mathfrak{R}_{\mathrm{i}}=d_{m} / 2+\left(f_{i}-0.5\right) D_{b} \cos \alpha_{0}
\end{aligned}
$$

where, $f_{i}$ and $f_{o}$ are the curvature coefficient of inner groove and outer groove respectively, $d_{m}$ is the pitch diameter.

According to the positional relationship and the Pythagorean theorem, the groove contact deformation equation can be obtained as follows:

$$
\begin{aligned}
& \left(A_{1 j}-X_{1 j}\right)^{2}+\left(A_{2 j}-X_{2 j}\right)^{2}=\Delta_{i j}^{2} \\
& X_{1 j}{ }^{2}+X_{2 j}{ }^{2}=\Delta_{o j}^{2}
\end{aligned}
$$


where, $\Delta_{o j}$ is the distance between the outer raceway groove curvature center and the final position of the ball center at $j$-ball, $\Delta_{i j}$ is the distance between the inner raceway groove curvature center and the final position of the ball center at $j$-ball,

$$
\begin{aligned}
& \Delta_{o j}=\left(f_{o}-0.5\right) D_{b}+\delta_{o j} \\
& \Delta_{i j}=\left(f_{i}-0.5\right) D_{b}+\delta_{i j}
\end{aligned}
$$

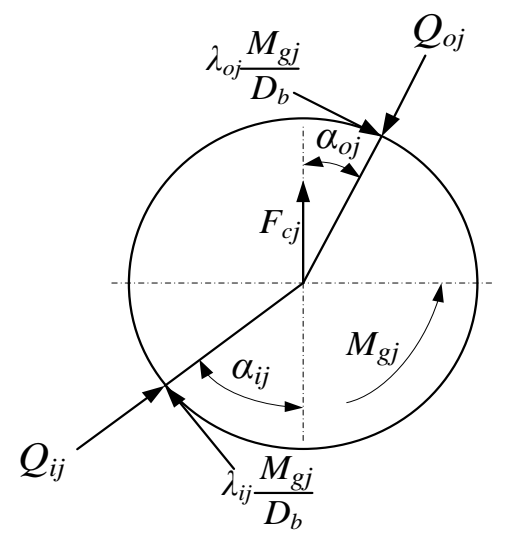

Fig. 3 Load on the j-th rolling element

According to the outer ring control theory, $\lambda_{i j}=0, \lambda_{o j}=2$ and the force situation of the $j$-th ball is shown in Fig. 3 , the equilibrium equations of the $j$-th rolling elements in the horizontal and vertical directions are expressed as follows:

$$
\begin{aligned}
& Q_{i j} \sin \alpha_{i j}-Q_{o j} \sin \alpha_{o j}-\frac{M_{g j}}{D_{b}}\left(\lambda_{i j} \cos \alpha_{i j}-\lambda_{o j} \cos \alpha_{o j}\right)=0 \\
& Q_{i j} \cos a_{i j}-Q_{o j} \cos a_{o j}-\frac{M_{g j}}{D_{b}}\left(\lambda_{i j} \sin a_{i j}-\lambda_{o j} \sin a_{o j}\right)=-F_{c j} \\
& Q_{i j}=K_{\mathrm{ij}} \delta_{i j}^{1.5} \\
& Q_{\mathrm{oj}}=K_{o j} \delta_{o j}^{1.5} \\
& F_{c j}=\frac{1}{2} m_{b} d_{m} \omega^{2}\left(\frac{\omega_{m}}{\omega}\right)_{j}^{2} \\
& M_{g j}=J\left(\frac{\omega_{r}}{\omega}\right)_{j}\left(\frac{\omega_{m}}{\omega}\right)_{j} \omega^{2} \sin \beta
\end{aligned}
$$

where, $F_{c j}$ is the centrifugal force, $m_{b}$ is the mass of rolling element, $\omega$ is the rotational speed, $\omega_{m}$ is the orbital speed of rolling element, $J$ is the mass moment of inertia of rolling element, $\omega_{r}$ is the speed of roller about its own axis, $\beta$ is the rolling element attitude angle, $Q_{i j}$ and $Q_{o j}$ are the contact loads at the inner and outer ring, respectively, $K_{i j}, K_{o j}$ are the load-deformation coefficients at the inner and outer rings, respectively.

According to the above force analysis of the force of the rolling element of bearings and the internal deformation relationship, the force balance equations of the entire bearing are obtained as follows: 


$$
\begin{aligned}
F_{a} & =\sum_{j=1}^{j=Z}\left(Q_{i j} \sin \alpha_{i j}-\frac{\lambda_{i j} M_{g j}}{D_{b}} \cos \alpha_{i j}\right) \\
F_{\mathrm{r}} & =\sum_{j=1}^{j=Z}\left(Q_{i j} \cos \alpha_{i j}-\frac{\lambda_{i j} M_{g j}}{D_{b}} \sin \alpha_{i j}\right) \cos \psi_{j} \\
M & =\sum_{j=1}^{j=Z}\left[\left(Q_{i j} \sin \alpha_{i j}-\frac{\lambda_{i j} M_{g j}}{D_{b}} \cos \alpha_{i j}\right) \Re_{i}+\frac{\lambda_{i j} M_{g j}}{D_{b}} \mathrm{r}_{i}\right] \cos \psi_{j}
\end{aligned}
$$

where, $\mathrm{Z}$ is the number of rolling elements.

\subsection{Bearing contact stiffness under multi-factor effect}

Taking into account the centrifugal effect, the gyroscopic moment and the axial and radial thermal deformation, a modified quasi-static model is obtained. Eqs. (7) (8), Eqs. (13) (14) and Eqs. (19) (21) are integrated into nonlinear equations, and the Newton-Raphson iteration method is used to solve the dynamic parameters, and then the contact loads between the rolling element and the rings are calculated, finally, the contact stiffness of the bearing is obtained, which can be written as follows:

$$
K_{\mathrm{c}}=\frac{d Q}{d \delta}=1.5 \Gamma_{j}^{-1}\left[\left(\frac{9}{2 \varepsilon_{j} R_{j}}\right)\left(\frac{1}{\pi k_{j} E^{\prime}}\right)^{2}\right]^{-1 / 3} Q_{j}^{1 / 3}
$$

where, $\Gamma$ is complete elliptic integral of the first kind, $\varepsilon$ is complete elliptic integral of the second kind, $R$ is the sum of the curvatures of the contact points of the two contacting objects in the main plane, $k_{j}$ is the ellipticity of the contact ellipse, $E^{\prime}$ is the equivalent elastic modulus, $K_{c}$ is the contact stiffness.

\subsection{Model verification}

In order to verify the accuracy of this model, a calculation example from reference (Huang et al.,2017) is adopted. The contact angle and the contact load of the bearing were calculated in the example, but the thermal effect and its influence were not considered in the reference. The main parameters of the bearing from literature (Huang et al.,2017) are shown in Tab. 1.

Table 1 Main parameters of the bearing

\begin{tabular}{ll}
\hline Parameter & Value \\
\hline Number of rolling elements & 16 \\
Rolling element diameter/mm & 7.90 \\
Bearing pitch diameter/mm & 54 \\
Original contact angle/ ${ }^{\circ}$ & 15 \\
Inner groove curvature coefficient & 0.52 \\
Outer groove curvature coefficient & 0.52 \\
Modulus of elasticity/GPa & 206 \\
Poisson's ratio & 0.3 \\
Radial load applied/N & 500 \\
Speed/r/min & 20000 \\
\hline
\end{tabular}


The comparative results between two models are given in Tab. 2 .

Table 2 Comparison of two calculation results

\begin{tabular}{|c|c|c|c|}
\hline parameter & Literature & This & Relative error \\
\hline Inner ring contact angle/ ${ }^{\circ}$ & 19 & 18.8 & $1.05 \%$ \\
\hline Outer ring contact angle $/^{\circ}$ & 13.4 & 12.9 & $3.73 \%$ \\
\hline Inner ring contact load/N & 91.7 & 96.7 & $5.45 \%$ \\
\hline Outer ring contact load/N & 135 & 127.2 & $5.78 \%$ \\
\hline
\end{tabular}

It can be seen from Tab. 2 that there is only slight difference between two sets of the contact parameters. The maximum percentage difference between the results is $5.78 \%$. The result of the proposed model is basically consistent with the results in the literature, which verifies the correctness of the model proposed in this paper.

\section{Film stiffness model of angular contact ball bearing considering temperature}

\subsection{Film thickness}

Based on the formula of point contact dimensionless minimum oil film thickness presented by Hamrock(Wen et al.,1992), the $H_{\min }$ is:

$$
H_{\min }=3.63 U^{0.68} G^{0.49} W^{-0.073}\left(1-e^{-0.68 k}\right)
$$

where, $U$ is the speed parameter, $G$ is the material parameter, $W$ is the load parameter, $k$ is the ellipticity. The expressions for them are:

$$
\begin{aligned}
& U=u_{\mathrm{s}} \eta / E^{\prime} R_{x} \\
& W=w / E^{\prime} R_{x}^{2} \\
& G=\alpha_{1} E^{\prime}
\end{aligned}
$$

where $\eta$ is the lubricant dynamic viscosity, $\alpha_{1}$ is the lubricant viscosity-pressure coefficient, $R_{x}$ is the equivalent curvature radius of the rolling element along $x$-direction, $w$ is the contact load, $u_{s}$ is the average speed.

Considering the influence of temperature on viscosity, Roelands (1966) viscosity-pressure formula is used as follows:

$$
\begin{aligned}
& \eta=\eta_{0} \exp \left\{\left(\ln \eta_{0}+9.67\right)\left[\left(1+p / p_{0}\right)^{z}\left(\frac{T-138}{T_{0}-138}\right)^{-1.1}-1\right]\right\} \\
& z=\frac{\alpha_{1}}{5.1 \times 10^{-9}\left(\ln \eta_{0}+9.67\right)}
\end{aligned}
$$

where, $\eta_{0}$ is the dynamic viscosity of lubricant under normal pressure, $p$ is the working pressure, $p_{0}$ is the atmospheric pressure, $T$ is lubricant temperature during operation, $T_{0}$ is the normal temperature, $\alpha_{1}$ is the viscous pressure coefficient. 
The minimum oil film thickness $h_{\min }$ between the rolling element and the inner and outer rings can be written as follows:

$$
h_{\min }=R_{\mathrm{x}} H_{\min }
$$

The parameters of film thickness are listed in Tab. 3 .

Table 3 parameters of bearing film thickness

\begin{tabular}{llll}
\hline parameter & value & parameter & value \\
\hline$k$ & $1 \sim 9.5$ & $G$ & $2500 \sim 5000$ \\
$u_{b} / \mathrm{m} / \mathrm{s}$ & $1 \sim 40$ & $W$ & $3.23 \times 10^{-4} \sim 1.94 \times 10^{-3}$ \\
$w / \mathrm{N}$ & $200 \sim 10^{4}$ & $U$ & $1.68 \times 10^{-11} \sim 6.74 \times 10^{-10}$ \\
\hline
\end{tabular}

\subsection{Film stiffness}

According to the definition of stiffness, the film stiffness of the bearing can be obtained by combining Eq. (25), (27) with (31), namely:

$$
K_{o i l}=\frac{d w}{d h_{\min }}
$$

\section{Comprehensive stiffness model}

In order to obtain the bearing stiffness closer to the actual situation, it is necessary to connect the contact stiffness with film stiffness in series to obtain the comprehensive stiffness. Therefore, the comprehensive stiffness between the $j$-th rolling element and the inner and outer rings is:

$$
\begin{aligned}
\frac{1}{K_{q i j}} & =\frac{1}{K_{c i j}}+\frac{1}{\left(K_{o i l}\right)_{i j}} \\
\frac{1}{K_{q o j}} & =\frac{1}{K_{c o j}}+\frac{1}{\left(K_{o i l}\right)_{o j}}
\end{aligned}
$$

where, $\left(K_{o i l}\right)_{i j}$ and $\left(K_{o i l}\right)_{o j}$ are the film stiffness between the $j$-th ball and the inner and outer rings respectively, $K_{q i j}$ and $K_{q o j}$ are the comprehensive stiffness between the $j$-th ball and the inner and outer rings, respectively.

According to the Harris' calculation formula of stiffness, the stiffness components in the axial and radial directions between the $j$-th ball and the inner and outer rings are:

$$
\begin{aligned}
& K_{a i j}=K_{q i j} \sin ^{2} \alpha_{i j} \\
& K_{a o j}=K_{q o j} \sin ^{2} \alpha_{o j} \\
& K_{r i j}=K_{q i j} \cos ^{2} \alpha_{i j} \\
& K_{r o j}=K_{q o j} \cos ^{2} \alpha_{o j}
\end{aligned}
$$


where, $K_{a i j}$ and $K_{r i j}$ are the axial and radial composite stiffness of the $j$-th ball and the inner ring, $K_{a o j}$ and $K_{r o j}$ are the axial and radial composite stiffness of the $j$-th ball and the outer ring, respectively.

For angular contact ball bearings, the entire comprehensive stiffness can be derived from the stiffness components between the balls and the inner and outer rings, which are calculated as:

$$
\begin{aligned}
& K_{a}=\sum_{j=1}^{j=Z} \frac{K_{a i j} K_{a o j}}{K_{a i j}+K_{a o j}} \\
& K_{r}=\sum_{j=1}^{j=Z} \frac{K_{r i j} K_{r o j}}{K_{r i j}+K_{r o j}} \cos ^{2}\left[\frac{2 \pi}{Z}(j-1)\right] \\
& K_{\theta}=\frac{d_{m}^{2}}{4} \sum_{j=1}^{j=Z} \frac{K_{a i j} K_{a o j}}{K_{a i j}+K_{a o j}} \cos ^{2}\left[\frac{2 \pi}{Z}(j-1)\right]
\end{aligned}
$$

where, $K_{a}, K_{r}$ and $K_{\theta}$ are the comprehensive axial stiffness, comprehensive radial stiffness and comprehensive angular stiffness, respectively.

\section{Calculation results and analysis}

In this paper, $\mathrm{H} 7013 \mathrm{C}$ angular contact ball bearing is taken as the research object, and the preloading method is positioning preloading. According to the above derived comprehensive stiffness model of angular contact ball bearing, the variation law of the comprehensive stiffness under the condition of multi-factor coupling is analyzed. The bearing structure and material parameters are shown in Tab.4.

The members of the research group measured the temperature rise of each component when the bearing speed was $0 \mathrm{r} / \mathrm{min} \sim 15000 \mathrm{r} / \mathrm{min}$ and compared it with the temperature obtained by simulation results. The results showed that the simulation model was effective (Zhang, 2019). On the basis, the temperature changes of each part of the bearing at 0r/min-24000r/min are obtained through simulation calculation, and the radial and axial thermal deformation of the bearing can be calculated by the formula.

Table 4 Basic parameters of angular contact ball bearing H7013C

\begin{tabular}{cc}
\hline Parameter & Values \\
\hline Inner ring diameter $/ \mathrm{mm}$ & 65 \\
Outer ring diameter/mm & 100 \\
Rolling element diameter $/ \mathrm{mm}$ & 8.73 \\
Number of rolling elements & 25 \\
Bearing width/ $\mathrm{mm}$ & 18 \\
Original contact angle/ & \\
Inner groove curvature coefficient & 15 \\
Outer groove curvature coefficient & 0.56 \\
Modulus of elasticity/GPa & 0.53 \\
Poisson's ratio & 206 \\
\hline
\end{tabular}

\subsection{The effect of speed on bearing temperature rise}

Fig. 4 shows the temperature rise of various parts of the bearing by simulation. It can be seen from the Fig. 4 that as the speed increases, the temperature rise of each part of the bearing gradually increases. At the same speed, the temperature rise of the inner ring is the largest, followed by that of the balls, and that of the outer ring is the smallest. This is mainly because the inner ring and the rotating shaft is interference mounted, and the temperature 
of the rotating shaft is directly transmitted to the inner ring, meanwhile, the heat from the rolling element is also transferred to the inner ring. The temperature rise of the outer ring mainly comes from the heat transfer of the rolling elements, while some of the heat is taken away by the cool system. Therefore, the outer ring temperature is the lowest.

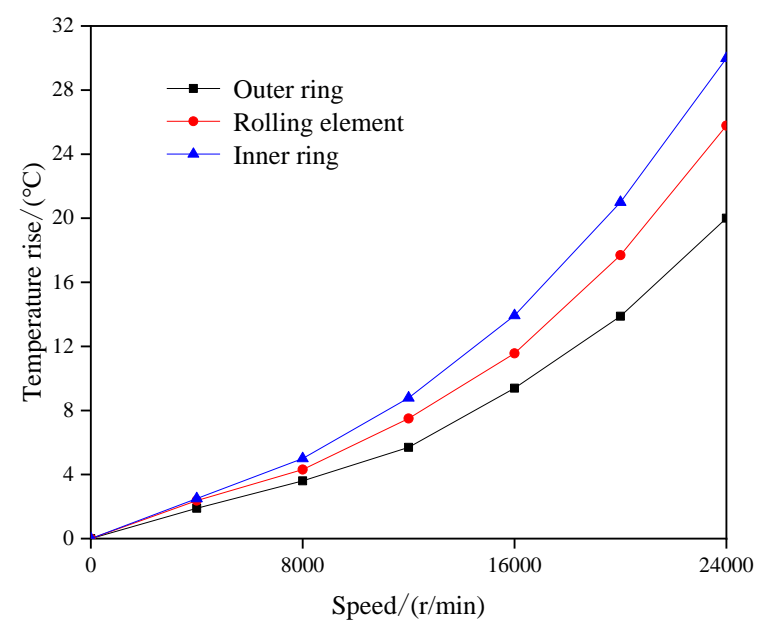

Fig. 4 Temperature rise of various bearing parts

\subsection{The effect of combined load on bearing contact stiffness}

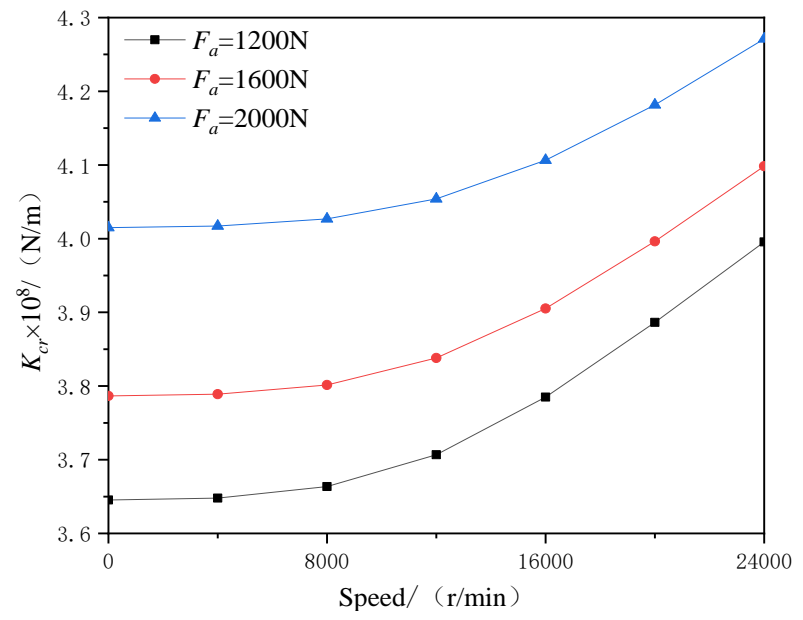

(a) Radial stiffness

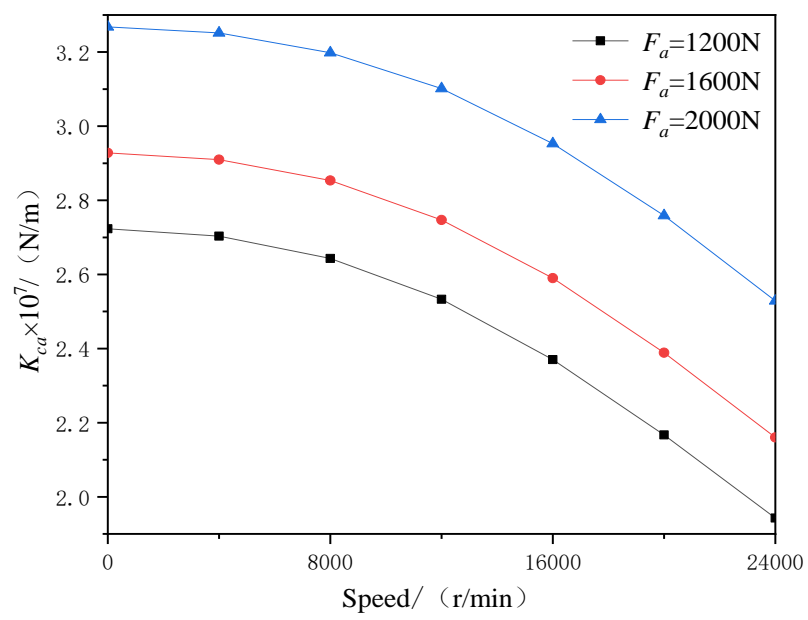

(b) Axial stiffness 


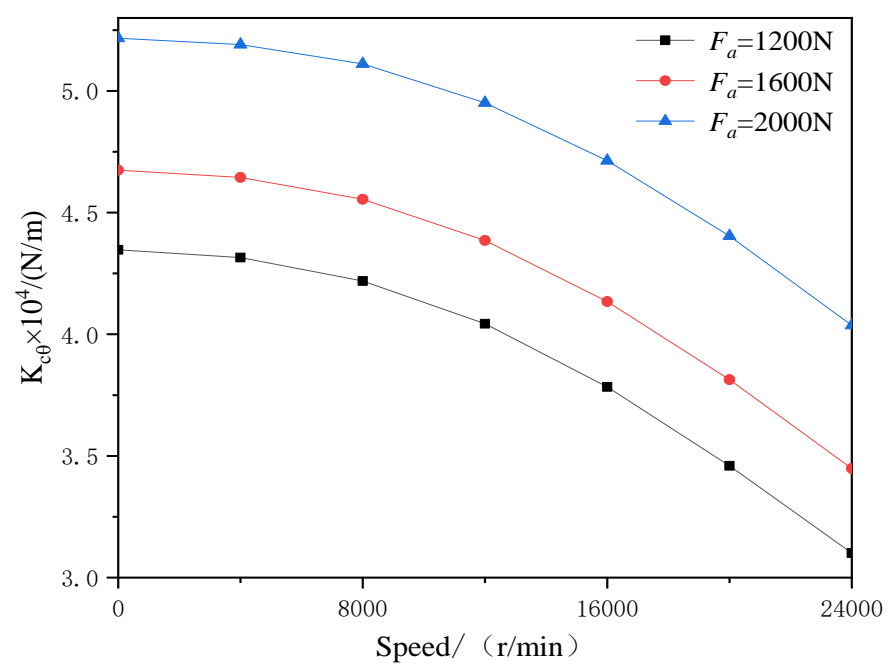

(c) Angular stiffness

Fig. 5 Bearing contact stiffness under different axial loads

Taking into account the influence of centrifugal force, gyroscopic torque and thermal deformation, the contact stiffness of the bearing is calculated. Fig. 5 shows the change of bearing contact stiffness under different axial loads when the radial load $F_{r}=100 \mathrm{~N}$. Fig.5(a), 5(b) and 5(c) respectively show the radial, axial and angular contact stiffness of the bearing. It can be seen from the Fig. 5 that as the speed increases, the radial contact stiffness gradually increases, while the axial and angular contact stiffness decreases. The radial contact stiffness increases, such phenomena are due to the fact that the positioning preloading method is adopted in the bearing, and the relative position of the inner and outer rings is restricted. When the bearing is running at high speed, part of the relative displacement will be converted into strain, which increases the axial force and radial stiffness. In addition, it can also be seen that as the axial load increases, the radial, axial and angular contact stiffness increase, which is due to the contact angle and contact load also increase as the axial load increases. This change trend is consistent with reference (Wang et al., 2010).

On the other hand, when the axial load $F_{a}=2000 \mathrm{~N}$, bearing contact stiffness under different radial loads are given in Fig.6. It can be seen from Fig. 6 that when the rotational speed is constant, as the radial load increases, the radial, axial and angular contact stiffness continuously reduced. This is because that with the increase of radial load, the number of balls in contact with inner ring gradually decreases, and the deformation of each contact ball increases, which results in the decrease of radial, axial and angular contact stiffness.

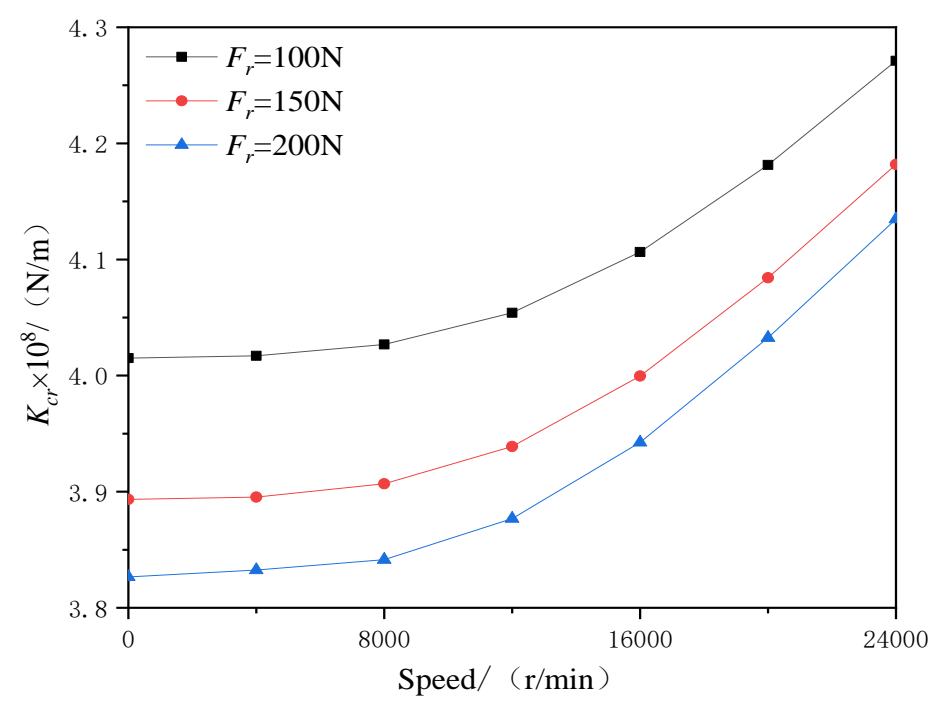

(a) Radial stiffness 


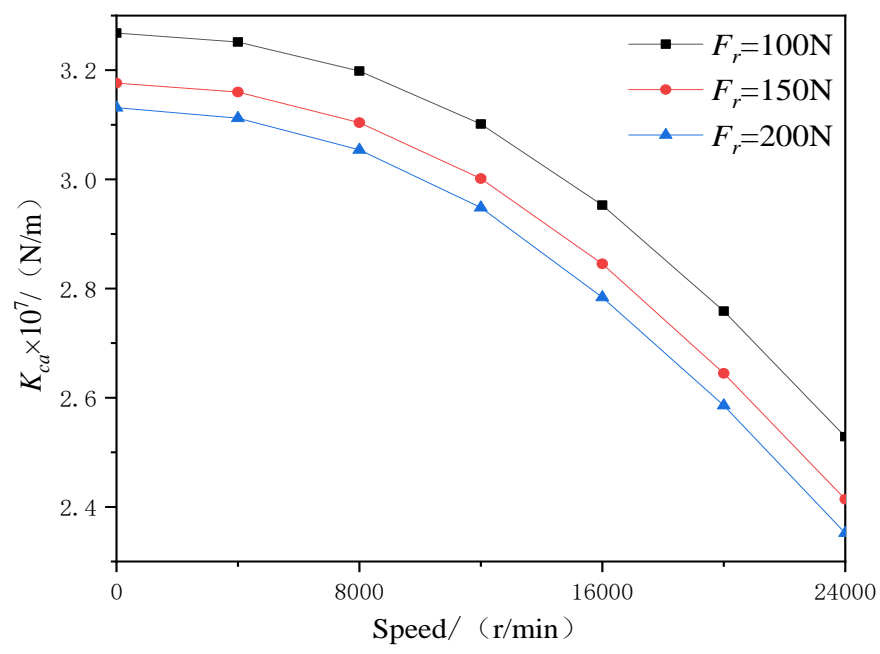

(b) Axial stiffness

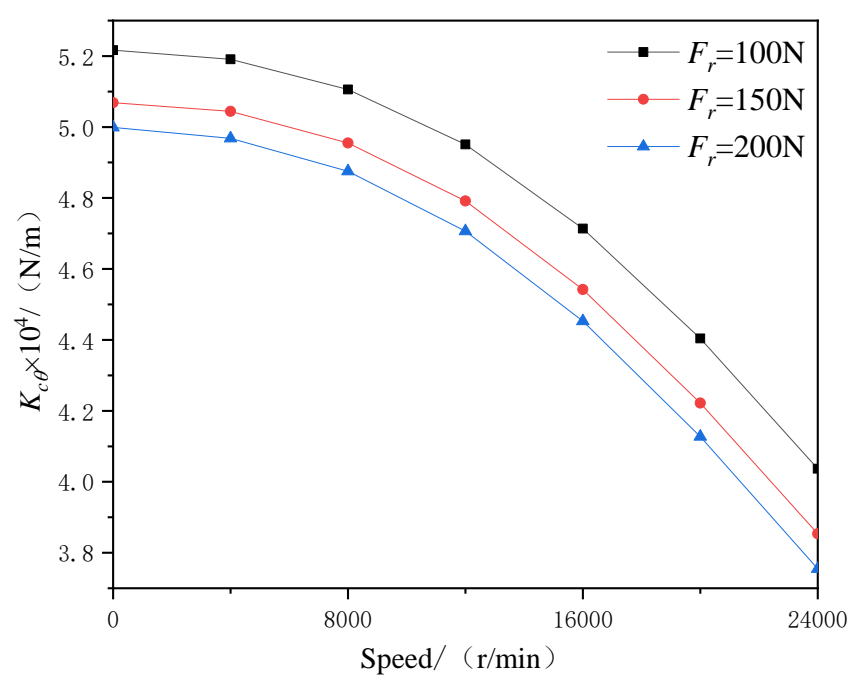

(c) Angular stiffness

Fig. 6 Bearing contact stiffness under different radial loads

\subsection{The influence of speed on the dynamic viscosity of lubricating oil}

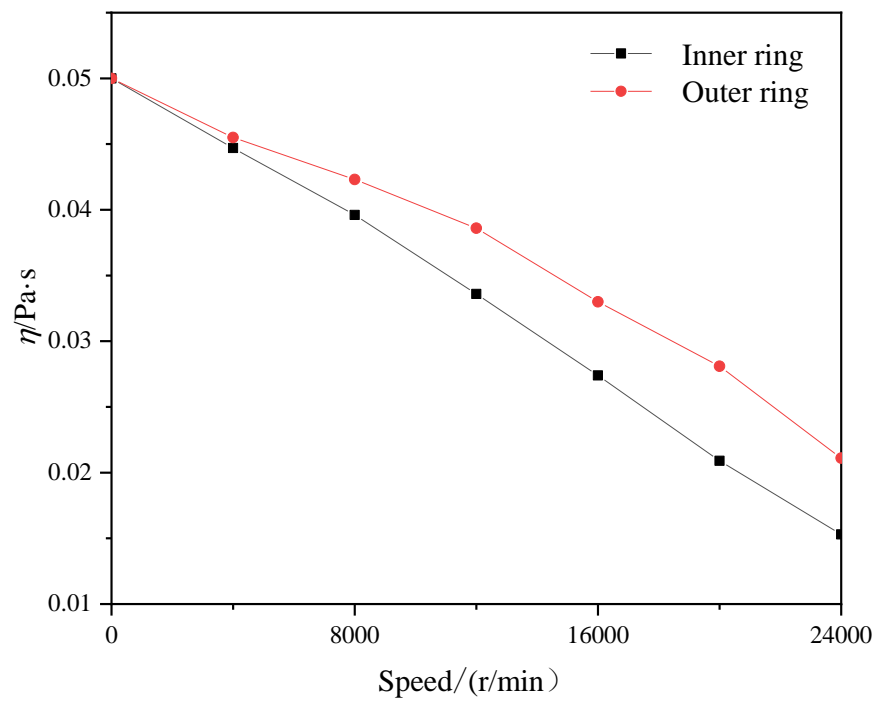

Fig. 7 Relationship between the dynamic viscosity and the speed 
According to Eq. (29), the dynamic viscosity considering the temperature rise can be obtained. In Fig. 7, as the bearing speed increases, the dynamic viscosity of the lubricating oil decreases due to the temperature rise. In addition, the dynamic viscosity of the lubricant between the inner ring and the balls decreases faster than that of the outer ring. The reason is that the temperature of the inner ring changes more drastically with the speed, so the dynamic viscosity decreases obviously.

\subsection{The influence of temperature on film stiffness}

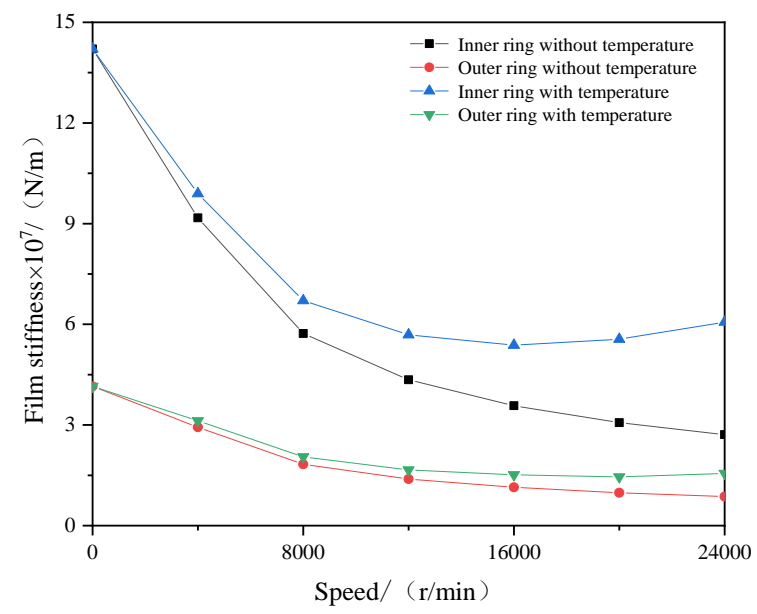

Fig. 8 The influence of speed on film stiffness.

Fig. 8 shows when $F_{a}=2000 \mathrm{~N}$ and $F_{r}=100 \mathrm{~N}$, the variation law of film stiffness with the increase in speed. In Fig.8, it is found that as the speed increases, the film stiffness decreases non-linearly. The reason for this phenomenon is that the increase of the speed is conductive to the formation of the elastohydrodynamic lubricant film, the minimum film thickness increases and the film stiffness reduces. This change trend is consistent with reference (Wu et al.,2014). It can also be seen that the film stiffness considering the temperature is larger than that without consideration of the temperature. Meanwhile, the effect of temperature on the dynamic viscosity is considered, the film stiffness of the bearing decrease firstly, when the speed reaches a certain value, the film stiffness increases. This is because the film stiffness decreases with the increase of the speed. At the same time, as the speed increases, the dynamic viscosity of the bearing decreases and the minimum film thickness decreases, and then the film stiffness increase. The inflection point can be seen in Fig. 8. This also shows that the bearing temperature change caused by the increase in speed cannot be ignored in the calculation of the film stiffness.

\subsection{The effect of combined load on the comprehensive stiffness}

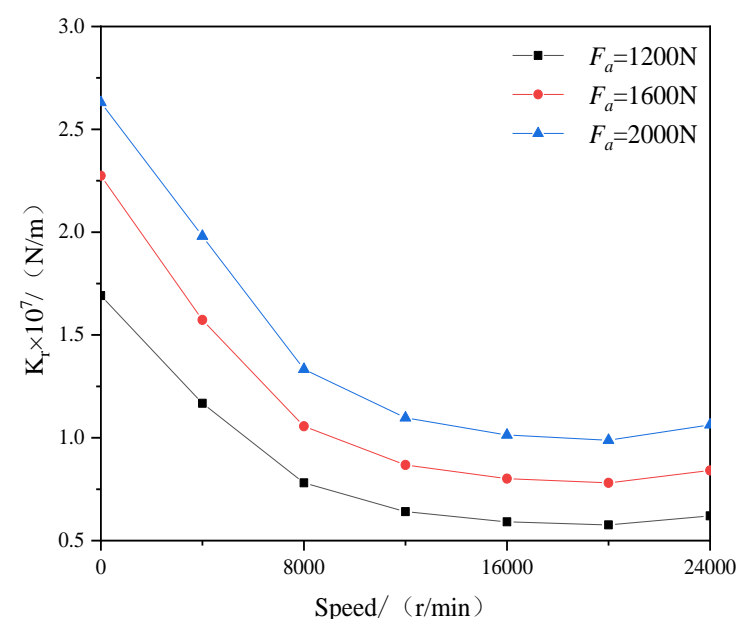

(a) Radial stiffness 


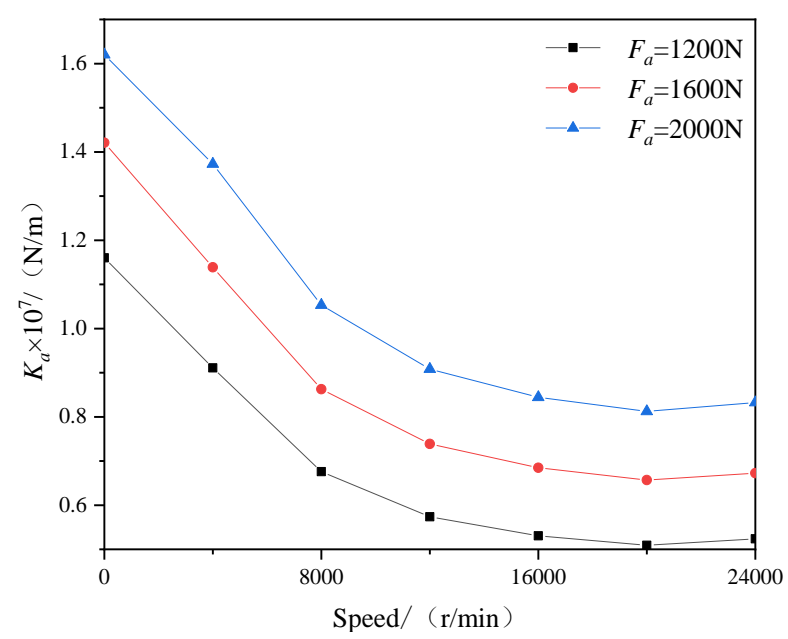

(b) Axial stiffness

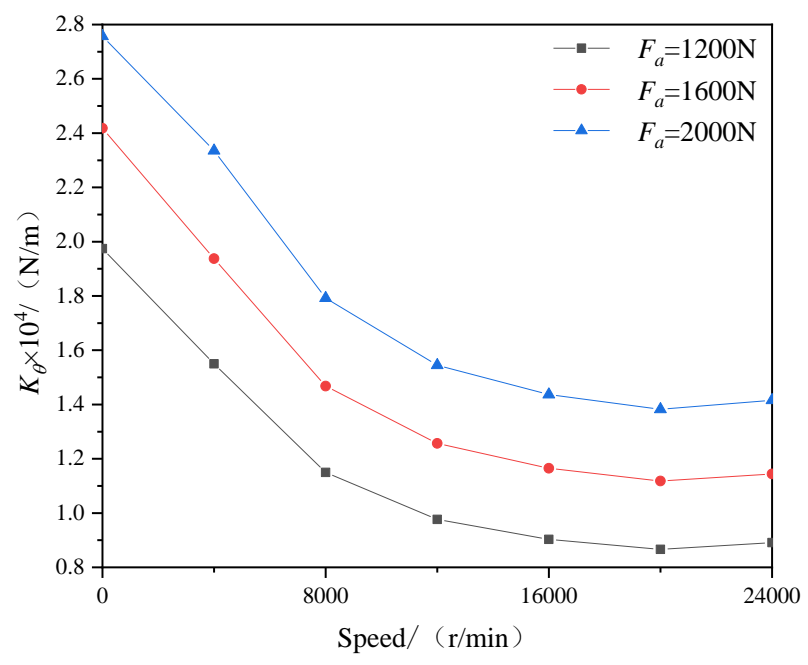

(c) Angular stiffness

Fig. 9 The effect of axial load on the comprehensive stiffness

As illustrated in Fig. 9, when $F_{r}=100 \mathrm{~N}$, with the increase of axial load, the comprehensive radial, axial and angular stiffness are increasing. This is due to the increase in axial load, the contact stiffness and film stiffness also increase, and the contact stiffness and film stiffness are connected in series, so the overall stiffness increases.

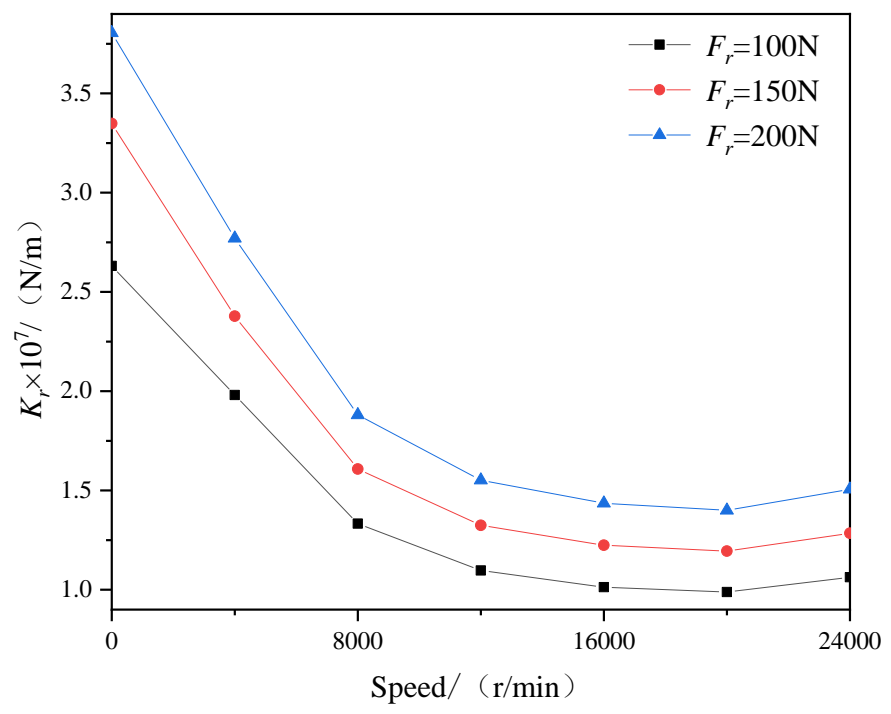

(a) Radial stiffness 


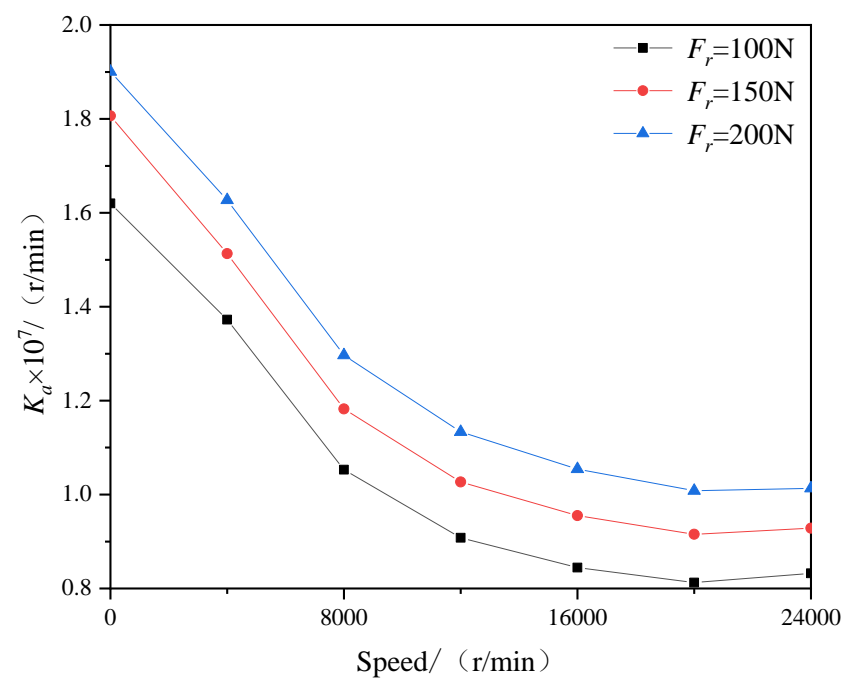

(b) Axial stiffness

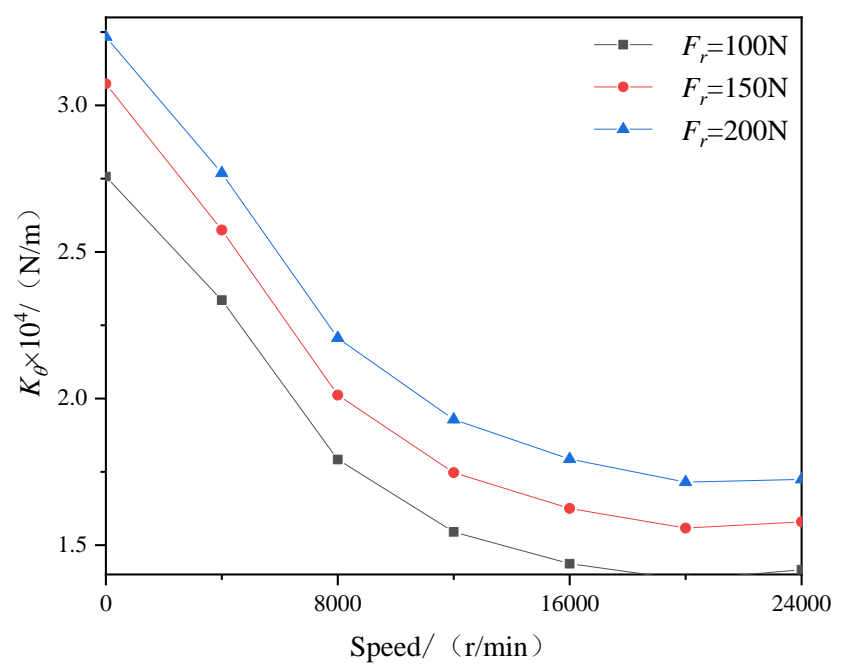

(c) Angular stiffness

Fig. 10 The influence of radial load on the comprehensive stiffness

On the other hand, Fig.10 shows the change of the comprehensive stiffness under different radial loads when $F_{a}=2000 \mathrm{~N}$. As the radial load increases from $100 \mathrm{~N}$ to $200 \mathrm{~N}$, the comprehensive stiffness continues to increase. Compared with Fig.6, it can be seen that variation of the comprehensive stiffness is different from that of the contact stiffness. This is because with the increase of radial load, the contact stiffness decreases, while the film stiffness increases, and the increase amplitude is greater than the decrease range of contact stiffness, so the comprehensive stiffness increases gradually.

\subsection{The effect of temperature on the comprehensive stiffness}

Fig. 11 shows the effect of temperature on the comprehensive stiffness under $F_{a}=2000 \mathrm{~N}$ and $F_{r}=100 \mathrm{~N}$. It can be seen from Fig. 11 that when the influence of temperature on the dynamic viscosity of the lubricant is unconsidered, the comprehensive radial, axial and angular stiffness decrease with the increase of speed. This is because the film stiffness and contact stiffness are in the same order of magnitude and show a downward trend. Under the action of these stiffness in series, the comprehensive stiffness decreases. Considering the influence of temperature on the dynamic viscosity, the comprehensive stiffness decreases with the increases of speed. However, when the speed in larger than $20000 \mathrm{r} / \mathrm{min}$, the comprehensive stiffness increases. This is because as the speed increases, the dynamic viscosity decreases, and the minimum oil film thickness decreases, and then film stiffness increases, so the comprehensive 
stiffness also increases. Moreover, it can also be seen that the comprehensive stiffness considering the temperature is larger than without consideration of the temperature.

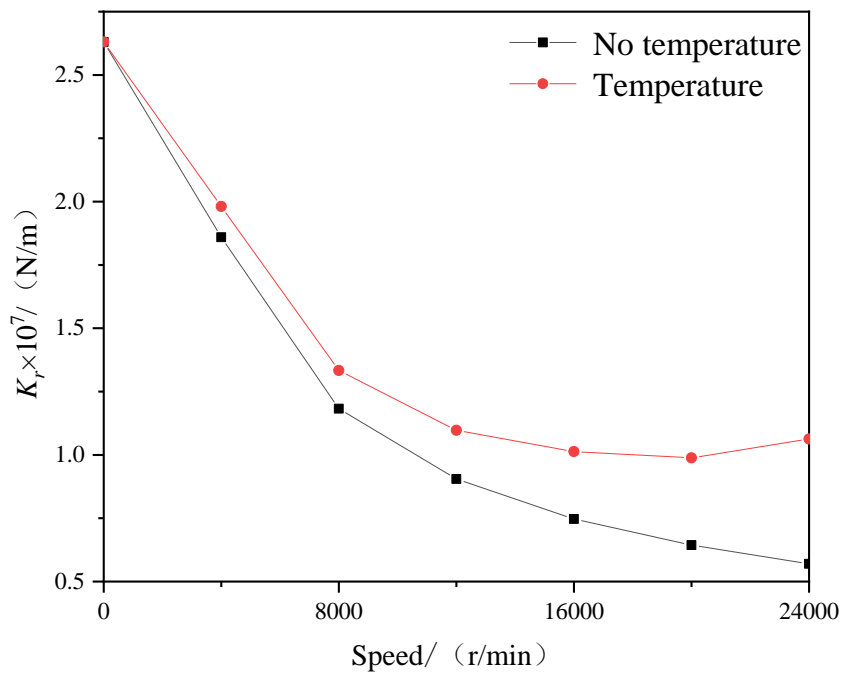

(a) Radial stiffness

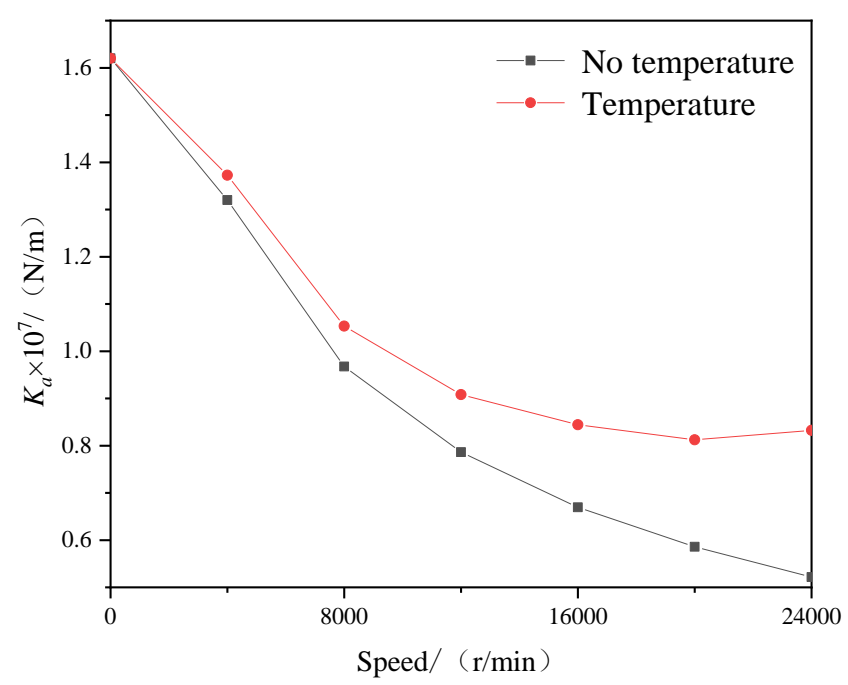

(b) Axial stiffness

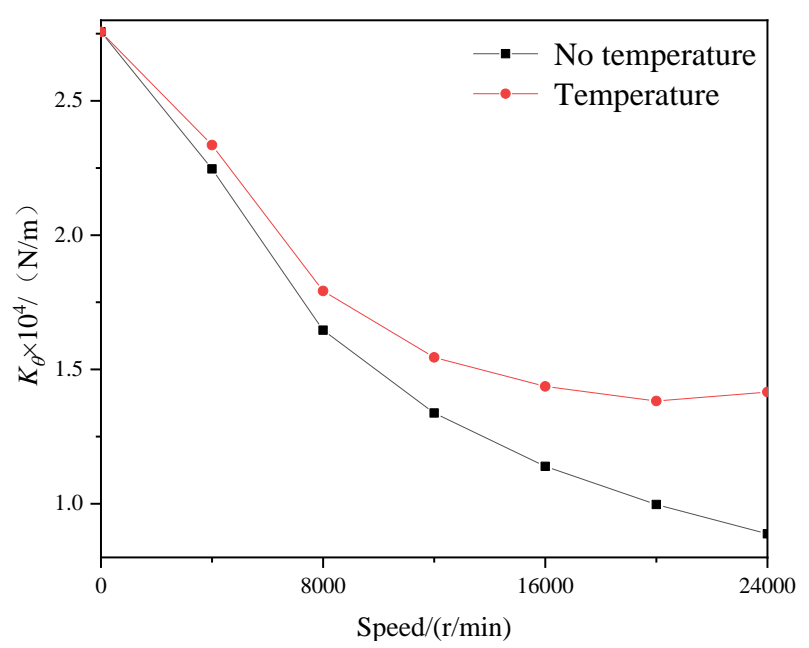

(c) Angular stiffness

Fig. 11 The effect of temperature on comprehensive stiffness 


\section{Conclusions}

In this paper, based on Jones' quasi-static model, considering the influence of coupling centrifugal force, gyroscopic torque and thermal deformation on the contact stiffness and the influence of temperature on the dynamic viscosity of lubricating oil at high speed, a modified calculation model of comprehensive stiffness is established. The change laws and influence factor of comprehensive stiffness of the bearing under the multi-factor coupling condition are investigated in detail. The following conclusions are obtained:

1. In the calculation of bearing contact stiffness under positioning preload, as the speed increases, the radial contact stiffness increases, while the axial stiffness and angular stiffness gradually decreases. With the increase of the axial load, the contact stiffness increases. With the increase of the radial load, the contact stiffness decreases.

2. As the speed increases, the film stiffness decreases. The film stiffness considering the temperature is larger than that without consideration of the temperature. Moreover, the influence of temperature rise on the dynamic viscosity is taken into account, the film stiffness increases as the speed reaches a certain value.

3. When the speed increases, the comprehensive radial, axial and angular stiffness of the bearing decrease. Considering the influence of temperature rise on the dynamic viscosity, the comprehensive stiffness decreases firstly and then increases. As the axial load increases, the comprehensive stiffness increases, and so does the increase of radial load.

\section{Acknowledgement}

This work was financially supported by the National Natural Science Foundation of China (Grant No. 51465035) and the Natural Science Foundation of Gansu Province of China (Grant No. 20JR5RA466).

\section{References}

Ali, N. J. and Garcfa, J. M., Experimental studies on the dynamic characteristics of rolling element bearings, Proceedings of the Institution of Mechanical Engineers, Part J: Journal of Engineering Tribology, Vol. 224, No. 7 (2010)

Dougdag, M., Ouali, M., Boucherit, H., Titouche, N. E. and Djaoui, M., An experimental testing of a simplified model of a ball bearing: stiffness calculation and defect simulation, Meccanica, Vol. 47, No. 2 (2012), pp. 335-354

Fawzi, M. A. and El-Saeidy., Time-Varying Total stiffness matrix of a rigid machine spindle-angular contact ball bearings assembly: Theory and Analytical/Experimental Verifications, Shock and Vibration, Vol. 18, No. 5 (2011).

Gupta, P. K., Advanced dynamics of rolling elements, Springer Verlag, Inc, New York (1984).

Hagiu, G. D. and Gafitanu, M., Dynamic characteristics of high speed angular contact ball bearings, Wear, Vol. 211, No. 1 (1997).

Harris, T. A., and Mindel, M. H., Rolling element bearing dynamics, Wear, Vol. 23, No.3 (1973), pp. 311-37.

Harris, T. A., Rolling Bearing Analysis, John Wiley and Sons, Inc, New York (1984).

Harris, T. A., Rolling bearing analysis, Wiley, New York (2001).

Huang, W. D., Gan, C. B., Yang, S. X. and Xu, L. H., Analysis on the stiffness of angular contact ball bearings and its effect on the critical speed of a high speed motorized spindle, Journal of Vibration and Shock, Vol. 36, No. 10(2017), pp. 19-25(in Chinese).

Jones, A. B., A general theory for elastically constrained ball and radial roller bearing under arbitrary load and speed conditions, Basic Eng, Vol. 82 (1960), pp. 309-320.

Jones, A. B., The Mathematical Theory of Rolling Elements Bearings, Mechanical Design and Systems Handbook, New York, Mc Graw-Hill (1966).

Jones, A. B. and McGrew, J., Rotor-bearing dynamics technology design guide, Part2: Ball Bearing (1978), pp. 1-62.

Lei, C. L., Li, F. H., Gong, B. R., Jia, X. B. A. and Gregory, C., An integrated model to characterize comprehensive stiffness of angular contact ball bearings, Mathematical Problems in Engineering (2020), pp. 1-12.

Li, J. H., Lei, C. L., Gong, B. R., Cui, P. and Jia, X. B., Modeling and analysis of the composite stiffness for angular contact ball bearings, Shock and Vibration (2020).

Noel, D., Ritou, M., Furet, B. and Le, L. S., Complete analytical expression of the stiffness matrix of angular contact 
ball bearings, Journal of Tribology, Vol. 135, No. 4 (2013).

Roelands, C. J. A., Correlational aspects of the viscosity-temperature-pressure relationship of Lubricating oils Durk, U R B, Groningen (1966).

Tang, Y. B., Research on the Mechanical Characteristics of Aero-engine Shaft High-speed Ball Bearings (Ph. D. Thesis), Nanjing University of Aeronautics and Astronautics (2005), Nanjing (in Chinese).

Wang, B. M., Mei, X. S., Hu, C. B. and Sun, J. R., Analysis on Dynamic Characteristics of Preloaded High-speed Angular Contact Ball Bearings, Bearing, No.5 (2010), 1-4+10 (in Chinese).

Wen, S. Z. and Yang, P. R., Elastohydrodynamic lubrication. Tsinghua University Press (1992).

Wu, M. X., Wu, W., Hu, J. B., Yuan, S. H. and Wei, C., Oil film stiffness calculation of high speed angular contact ball bearing considering spinning. Journal of Vibration and Shock, Vol. 33, No. 10 (2014), pp. 38-42 (in Chinese).

Xiong, W. L., Zhao, Z. S., Zhou, Y., Lv, L. and Hou, Z. Q., Research on dynamic stiffness of ball bearings considering ferrule deformation and elastohydrodynamic lubrication. Chinese Journal of Mechanical Engineering, Vol. 26, No. 11 (2015), pp. 1421-1428(in Chinese).

Yang, Z. H., Chen, H. and Yu, T. X., Effects of rolling bearing configuration on stiffness of machine tool spindle, Proceedings of the Institution of Mechanical Engineers, Vol. 232, No. 5 (2018).

Zhang, J. H., Fang, B., Hong, J., Wan, S. K. and Zhu, Y. S., A general model for preload calculation and stiffness analysis for combined angular contact ball bearings, Journal of Sound and Vibration, Vol. 411 (2017).

Zhang, J. H., Fang, B., Zhu, Y. S, Yan, K. and Hong, J., Investigation of the load distribution and stiffness characteristic of ball bearing under ball-raceway separation condition, Chinese Journal of Mechanical Engineering, Vol. 56, No. 9 (2020), pp. 73-83 (in Chinese).

Zhang, Y. B., Study on thermal characteristics of multi-parameter affected of high-speed motorized spindle, Lanzhou University of Technology, (2019) Lanzhou (in Chinese). 